\title{
A note on upper bound formulations in limit analysis
}

\author{
J. J. Muñoz ${ }^{1, * \dagger}$, A. Huerta ${ }^{1}$, J. Bonet ${ }^{2}$ and J. Peraire ${ }^{3}$ \\ ${ }^{1}$ Dep. Applied Mathematics III, LaCàN, Univ. Poitècnica de Catalunya (UPC), Barcelona, Spain \\ ${ }^{2}$ Civil and Computational Engineering Centre, School of Engineering, University of Wales, Swansea, UK \\ ${ }^{3}$ Department of Aeronautics and Astronautics, Massachusetts Institute of Technology (MIT), Cambridge, MA, USA
}

\begin{abstract}
SUMMARY
In this paper, we study some recent formulations for the computation of upper bounds in limit analysis. We show that a previous formulation presented by the authors does not guarantee the strictness of the upper bound, nor does it provide a velocity field that satisfies the normality rule everywhere. We show that these deficiencies are related to the quadrature employed for the evaluation of the dissipation power. We derive a formulation that furnishes a strict upper bound of the load factor, which in fact coincides with a formulation reported in the literature. From the analysis of these formulations, we propose a post-process, which consists in computing exactly the dissipation power for the optimum upper bound velocity field. This post-process may further reduce the strict upper bound of the load factor in particular situations. Finally, we also determine the quadratures that must be used in the elemental and edge gap contributions, so that they are always positive and their addition equals the global bound gap. Copyright (C) 2012 John Wiley \& Sons, Ltd.
\end{abstract}

Received 5 February 2010; Accepted 19 January 2011

KEY WORDS: limit analysis; bounds; optimisation; numerical integration

\section{INTRODUCTION}

Lower (upper) bounds of the load factor $\lambda$ in limit analysis are obtained by constructing discrete spaces of the velocity and stress fields, $v$ and $\sigma$, respectively, which are statically (kinematically) admissible. Although there is a common agreement in the choice of statically admissible spaces, several options have been reported when designing kinematically admissible spaces that yield upper bounds of $\lambda$. The objective of this paper is to study the properties of two proposed upper bound formulations, and suggest potential improvements.

Kinematically admissible spaces were originally derived by employing piecewise linear and continuous velocity fields. Although some recent (mixed) formulations have been proposed resorting to continuous velocity fields $[1,2]$, the incompressibility constraints in the velocities for some plasticity criteria does not allow them to furnish tight upper bounds of $\lambda$, unless some specific mesh arrangements are used. To improve the accuracy of these values, velocity discontinuities were originally added in [3] and [4]. These discontinuities were generalised in [5] and [6], and are currently widely exploited [7-12]. Among these references, the formulation in [6,9] considers some additional velocity variables at the edges, which guarantee the strictness of the upper bound, whereas the more recent article [11] uses quadratic discontinuous velocity fields, which are subjected to some conditions that guarantee its strictness. We also mention the related recent article [13], where the upper bounds of the load factor for multibody structures with frictional contact conditions between them are computed using a mixed linear complementary problem.

\footnotetext{
*Correspondence to: J. J. Muñoz, Dep. Applied Mathematics III, LaCàN, Univ. Poitècnica de Catalunya (UPC), Barcelona, Spain.

†E-mail: j.munoz@upc.edu 
On the other hand, the formulations in [8] and [7,12], consider just a discontinuous velocity field with no further restrictions. The former obtains this field by collapsing a triangular element to a line, whereas the latter directly introduces a linear velocity jumps. The aim of the present paper is to study the strictness and behaviour of these two simpler upper bound formulations reported in [8] and $[7,12]$, and introduce some improvements in the computation of the limit load factor.

After stating the primal and dual forms of the non-discretised problem, we discetise it and analyse the strictness of the upper bound formulation in terms of the quadrature employed for evaluating the dissipation power and the conditions that the problems include. This analysis is also extended to the expression of elemental and edge bound gap contributions.

\section{NON-DISCRETISED PROBLEM}

\subsection{Primal problem}

We will assume that the failure mechanism of a body $\Omega$ is not necessarily continuous. Hence, in addition to a continuous deformation, we also consider a failure mechanism involving a set of discontinuities at some internal boundaries $\Gamma$ of $\Omega$. The body is subjected to surface loads $\lambda g$ on the Neumann boundary $\Gamma_{N}$, and body loads $\lambda \boldsymbol{f}$, with $\lambda$ the unknown limit load factor. The lower bound theorem [14] states that the exact limit load is in this case computed as the following primal (maximisation) problem:

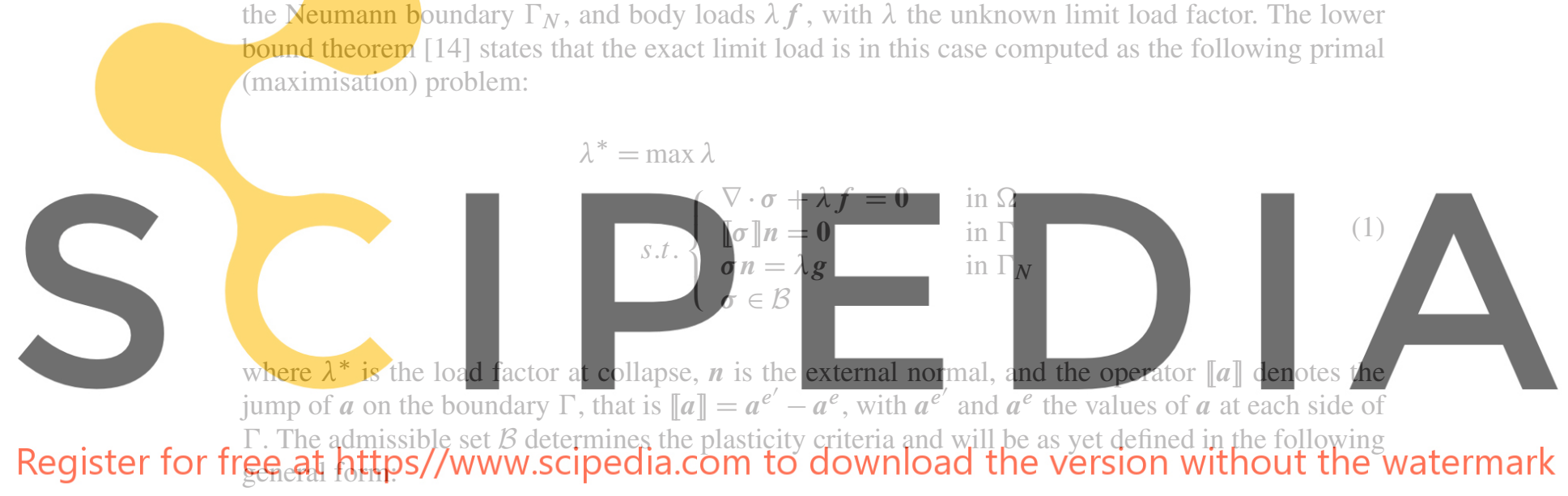

$$
\mathcal{B}:=\{\sigma \mid f(\sigma) \leqslant 0\} .
$$

\subsection{Saddle-point problem}

We will rewrite the maximisation problem in (1) as a saddle-point problem by using the following definitions:

$$
\begin{aligned}
a(\boldsymbol{v}, \boldsymbol{\sigma}) & :=\int_{\Omega} \boldsymbol{\varepsilon}(\boldsymbol{v}): \boldsymbol{\sigma} \mathrm{d} \Omega \\
b(\boldsymbol{v}, \boldsymbol{\sigma}) & :=\int_{\Gamma} \llbracket \boldsymbol{v} \rrbracket \cdot \boldsymbol{\sigma} \boldsymbol{n} \mathrm{d} \Gamma=\int_{\Gamma} \llbracket \boldsymbol{v} \rrbracket \bar{\otimes} \boldsymbol{n}: \boldsymbol{\sigma} \mathrm{d} \Gamma \\
\ell(\boldsymbol{v}) & :=\int_{\Omega} \boldsymbol{v} \cdot \boldsymbol{f} \mathrm{d} \Omega+\int_{\partial \Omega} \boldsymbol{v} \cdot \boldsymbol{g} \mathrm{d} \Gamma,
\end{aligned}
$$

where $\boldsymbol{\varepsilon}(\boldsymbol{v})=\frac{1}{2}\left(\nabla \boldsymbol{v}+(\nabla \boldsymbol{v})^{T}\right)$, and the operator $\bar{\otimes}$ is a symmetrised dyadic product: $\boldsymbol{a} \bar{\otimes} \boldsymbol{b}=$ $\frac{1}{2}(\boldsymbol{a} \otimes \boldsymbol{b}+\boldsymbol{b} \otimes \boldsymbol{a})$. The bilinear form $b(\boldsymbol{v}, \boldsymbol{\sigma})$ represents the internal power at the discontinuities $\Gamma$, $a(\boldsymbol{v}, \boldsymbol{\sigma})$ represents the internal power in $\Omega$, and the linear form $\ell(\boldsymbol{v})$ is the power of the external forces. On the boundary $\Gamma$, the vector $\boldsymbol{n}$ is the normal pointing from side $e$ towards side $e^{\prime}$ of the discontinuity, and $\boldsymbol{\sigma} \boldsymbol{n}=\frac{1}{2}\left(\boldsymbol{\sigma}^{e}+\boldsymbol{\sigma}^{e^{\prime}}\right) \boldsymbol{n}=\boldsymbol{\sigma}^{e} \boldsymbol{n}=\boldsymbol{\sigma}^{e^{\prime}} \boldsymbol{n}$, where the last two equalities hold due to the second condition in (1). 
By using the definitions in (3), and noting that the second condition in (1) also implies that $\llbracket \boldsymbol{\sigma} \boldsymbol{n} \cdot \boldsymbol{v} \rrbracket=\boldsymbol{\sigma} \boldsymbol{n} \cdot \llbracket \boldsymbol{v} \rrbracket$, the maximisation problem in (1) may be recasted as,

$$
\begin{aligned}
& \lambda^{*}=\max \lambda \\
& \text { s.t. }\left\{\begin{array}{l}
a(\boldsymbol{v}, \boldsymbol{\sigma})+b(\boldsymbol{v}, \boldsymbol{\sigma})=\lambda \ell(\boldsymbol{v}), \quad \forall \boldsymbol{v} \in \mathcal{V}, \\
\boldsymbol{\sigma} \in \mathcal{B}
\end{array}\right.
\end{aligned}
$$

with $\mathcal{V}$ the set of velocity functions with integrable bounded jumps on $\Gamma$ and integrable first derivatives on $\Omega$. By using this notation, the value of $\lambda^{*}$ in (1) may be also obtained as the following saddle-point problem:

$$
\begin{aligned}
\lambda^{*} & =\min _{\boldsymbol{v} \in \mathcal{V}} \max _{\boldsymbol{\sigma} \in \mathcal{B}}(\lambda+a(\boldsymbol{v}, \boldsymbol{\sigma})+b(\boldsymbol{v}, \boldsymbol{\sigma})-\lambda \ell(\boldsymbol{v})) \\
& =\min _{\substack{\boldsymbol{v} \in \mathcal{V} \\
\ell(\boldsymbol{v})=1}} \max _{\boldsymbol{\sigma} \in \mathcal{B}}(a(\boldsymbol{v}, \boldsymbol{\sigma})+b(\boldsymbol{v}, \boldsymbol{\sigma}))=a\left(\boldsymbol{v}^{*}, \boldsymbol{\sigma}^{*}\right)+b\left(\boldsymbol{v}^{*}, \boldsymbol{\sigma}^{*}\right),
\end{aligned}
$$

with $v^{*}$ and $\sigma^{*}$ some optimum velocity and stress fields that the collapse mechanism engenders.

\subsection{Dual problem and normality rule}

By defining the dissipation power $D(v)$ as,

$$
D(v):=\max _{\sigma \in \mathcal{B}}(a(v, \sigma)+b(v, \sigma)),
$$
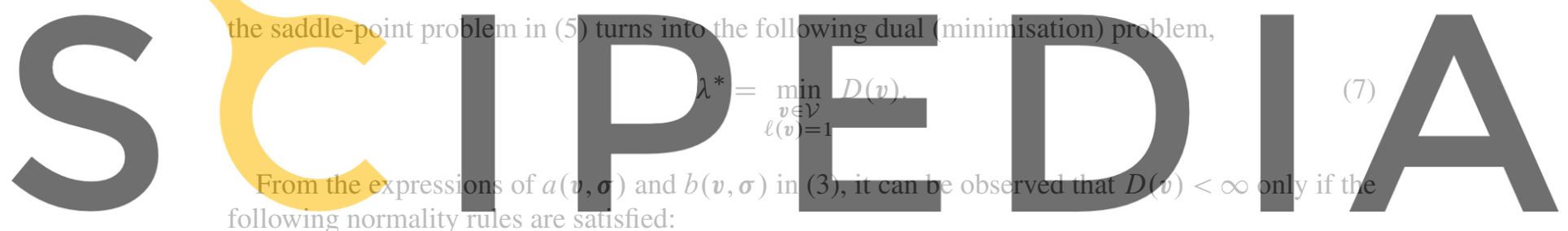

Register for free at https//www.scipedia.comf to download the version without the watermark

$$
\llbracket v \rrbracket \bar{\otimes} n=\gamma_{n} d,
$$

with $\gamma \geqslant 0$ and $\gamma_{n} \geqslant 0$ the plastic multipliers for the strain rates and the velocity jumps, respectively, and $\boldsymbol{d}$ a vector belonging to the sub-gradient of $f(\boldsymbol{\sigma})$, defined as $\partial f(\boldsymbol{\sigma})=\left\{\boldsymbol{d} \mid \boldsymbol{d}:\left(\boldsymbol{\sigma}-\boldsymbol{\sigma}^{*}\right) \geqslant\right.$ $\left.f(\sigma)-f\left(\sigma^{*}\right) \forall \sigma^{*}\right\}$. Wherever $f(\sigma)$ is differentiable, $\partial f(\sigma)=\frac{\partial f(\sigma)}{\partial \sigma}$. The normality conditions can be alternatively expressed as,

$$
\begin{aligned}
\boldsymbol{\varepsilon}(\boldsymbol{v}) & \in \mathcal{D} \\
\llbracket \boldsymbol{v} \rrbracket \bar{\otimes} \boldsymbol{n} & \in \mathcal{D}
\end{aligned}
$$

with $\mathcal{D}:=\{\boldsymbol{\varepsilon} \mid \boldsymbol{\varepsilon}=\gamma \partial f(\boldsymbol{\alpha})\}$ an admissible set for the strain rates and velocity jumps. For the yield functions that define the von Mises and Mohr-Coulomb criteria, the set $\mathcal{D}$ is defined by $[15,16]$ :

$$
\begin{aligned}
& \mathcal{D}_{V M}=\{\boldsymbol{\varepsilon} \mid \operatorname{tr}(\boldsymbol{\varepsilon})=0\} \\
& \mathcal{D}_{M C}=\left\{\boldsymbol{\varepsilon} \mid \operatorname{tr}(\boldsymbol{\varepsilon}) \geqslant\left(\left|\varepsilon_{1}\right|+\left|\varepsilon_{2}\right|+\left|\varepsilon_{3}\right|\right) \sin \phi\right\}
\end{aligned}
$$

whereas the velocity jumps must belong to the following admissible sets:

$$
\begin{aligned}
& \mathcal{D}_{V M}(\llbracket v \rrbracket)=\{\llbracket v \rrbracket \mid \llbracket v \rrbracket \cdot n=0\} \\
& \mathcal{D}_{M C}(\llbracket v \rrbracket)=\{\llbracket v \rrbracket \mid \llbracket v \rrbracket \cdot n \geqslant\|\llbracket v \rrbracket\| \sin \phi\}
\end{aligned}
$$

Note that although the previous condition for von Mises is linear, the one for Mohr-Coulomb is not, but both sets are convex. 
Assuming that the strain rates and velocity jumps are kinematically admissible, the dissipation power can be expressed solely as a function of the kinematic quantities as [16]:

$$
D(\boldsymbol{v})=\max _{\boldsymbol{\sigma} \in \mathcal{B}}\left(\int_{\Omega} \boldsymbol{\varepsilon}(\boldsymbol{v}): \boldsymbol{\sigma} \mathrm{d} \Omega+\int_{\Gamma} \boldsymbol{n} \bar{\otimes} \llbracket \boldsymbol{v} \rrbracket: \boldsymbol{\sigma} \mathrm{d} \Gamma\right)=\int_{\Omega} k(\boldsymbol{\varepsilon}(\boldsymbol{v})) \mathrm{d} \Omega+\int_{\Gamma} k_{n}(\llbracket \boldsymbol{v} \rrbracket) \mathrm{d} \Gamma=: \bar{D}(\boldsymbol{v}) .
$$

The explicit expressions of functions $k$ and $k_{n}$ for von Mises and 2D Mohr-Coulomb plastic criteria are given in Table I. We point out that $k_{n}(\llbracket v \rrbracket)$ is for Mohr-Coulomb criteria linear, whereas for von Mises is non-linear but convex.

If the dual problem is written in terms of $\bar{D}(\boldsymbol{v})$, instead of the definition of $D(\boldsymbol{v})$ in (6), the optimisation problem must be complemented with the membership constraints in (8), that is, Equation (7) turns into,

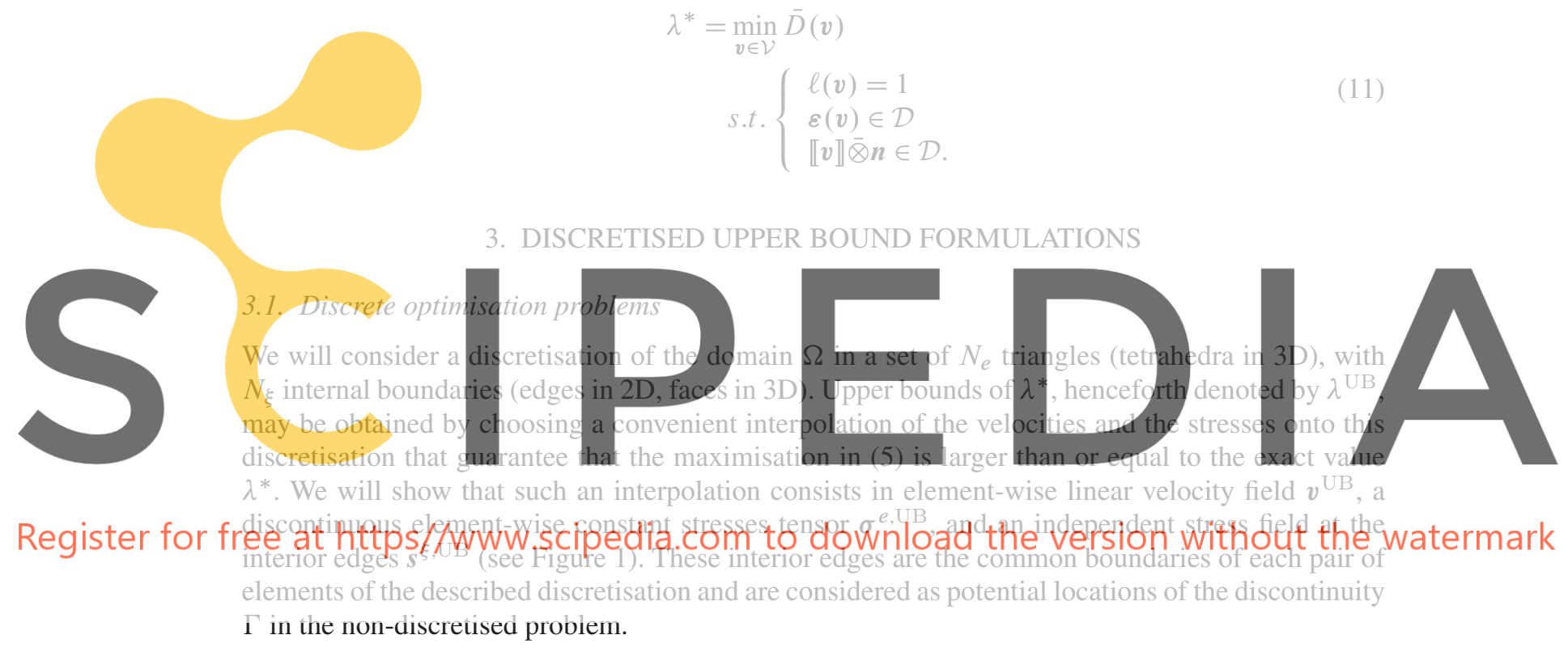

Table I. Material functions $k$ and $k_{n}$ in Equation (10) for von Mises and Mohr-Coulomb plastic criteria.

\begin{tabular}{lcc}
\hline & von Mises & Mohr-Coulomb \\
\hline$k(\boldsymbol{\varepsilon}(\boldsymbol{v}))$ & $\sqrt{\frac{2}{3}} \sigma_{Y}\|\boldsymbol{\varepsilon}(\boldsymbol{v})\|$ & $\frac{c}{\tan \phi} \operatorname{tr}(\boldsymbol{\varepsilon}(\boldsymbol{v}))$ \\
$k_{n}(\llbracket \boldsymbol{v} \rrbracket)$ & $\frac{\sigma_{Y}}{\sqrt{3}}\|\llbracket \boldsymbol{v} \rrbracket\|$ & $\frac{c}{\tan \phi} \llbracket v \rrbracket \cdot \boldsymbol{n}$ \\
\hline
\end{tabular}

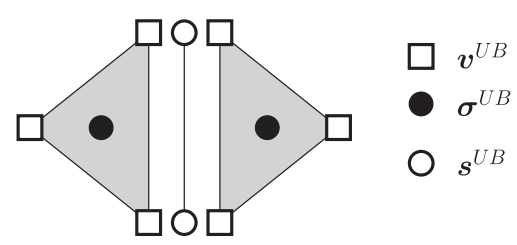

Figure 1. Velocity and stress discrete fields for the upper bound problem: linear velocities which are discontinuous at triangle internal edges $\left(v^{\mathrm{UB}}\right)$, constant stresses within each element $\left(\sigma^{\mathrm{UB}}\right)$, and linear stresses at the interior edges $\left(s^{\mathrm{UB}}\right)$. 
3.1.1. Primal optimisation problem. Let us first introduce the discrete counterparts of the forms $a(\boldsymbol{v}, \boldsymbol{\sigma}), b(\boldsymbol{v}, \boldsymbol{\sigma})$ and $\ell(\boldsymbol{v})$ in (3) as,

$$
\begin{aligned}
a\left(\boldsymbol{v}^{\mathrm{UB}}, \boldsymbol{\sigma}^{\mathrm{UB}}\right) & :=\sum_{e=1}^{N_{e}} a^{e}\left(\boldsymbol{v}^{e, \mathrm{UB}}, \boldsymbol{\sigma}^{e, \mathrm{UB}}\right) \\
b\left(\boldsymbol{v}^{\mathrm{UB}}, \boldsymbol{s}^{\mathrm{UB}}\right) & :=\sum_{\xi=1}^{N_{\xi}} b^{\xi}\left(\boldsymbol{v}^{\xi, \mathrm{UB}}, \boldsymbol{s}^{\xi, \mathrm{UB}}\right) \\
F\left(\boldsymbol{v}^{\mathrm{UB}}\right) & :=\sum_{e=1}^{N_{e}}\left(\int_{\Omega^{e}} \boldsymbol{v}^{\mathrm{UB}} \cdot \boldsymbol{f} \mathrm{d} \Omega+\int_{\Gamma_{N}} \boldsymbol{v}^{\mathrm{UB}} \cdot \boldsymbol{g} \mathrm{d} \Gamma\right) .
\end{aligned}
$$

Here, the bilinear forms $a^{e}\left(\boldsymbol{v}^{e, \mathrm{UB}}, \boldsymbol{\sigma}^{e, \mathrm{UB}}\right)$ and $b^{\xi}\left(\boldsymbol{v}^{\xi, \mathrm{UB}}, \boldsymbol{s}^{\xi, \mathrm{UB}}\right)$ are the internal power at each element and internal edge, respectively, and are explicitly given by,

$$
\begin{aligned}
& a^{e}\left(v^{e, \mathrm{UB}}, \sigma^{e, \mathrm{UB}}\right):=\int_{\Omega^{e}} \varepsilon\left(v^{e, \mathrm{UB}}\right): \sigma^{e, \mathrm{UB}} \mathrm{d} \Omega, \\
& b^{\xi}\left(v^{\xi, \mathrm{UB}}, s^{\xi, \mathrm{UB}}\right):=\int_{\Gamma^{\xi}} \llbracket v^{\xi, \mathrm{UB}} \rrbracket \bar{\otimes} n^{\xi}: s^{\xi, \mathrm{UB}} \mathrm{d} \Gamma .
\end{aligned}
$$

The value of $\lambda^{\mathrm{UB}}$ is then computed by particularising the maximisation problem in (4) to the mentioned discrete spaces:

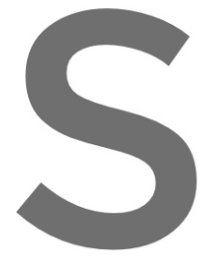

$$
\lambda^{\mathrm{UB}}=\max \lambda
$$
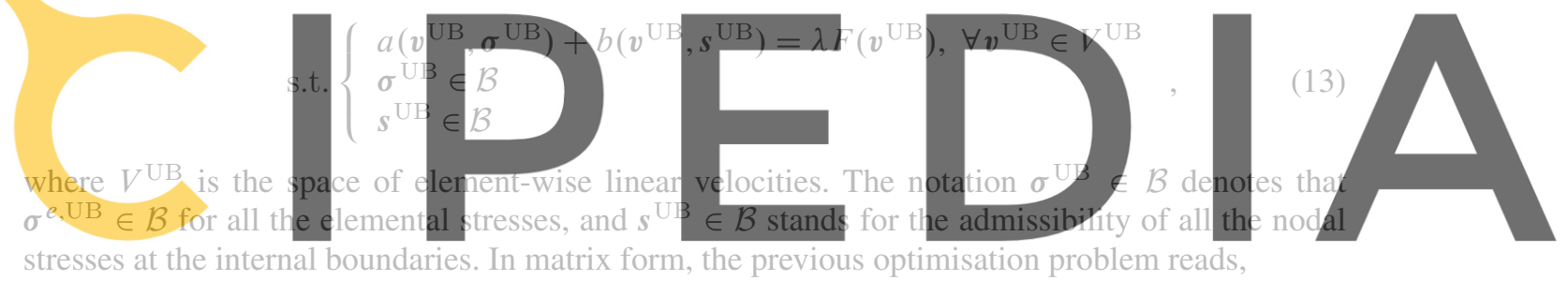

Register for free at https//www.seipedira.com to download the version without the watermark

$$
\text { s.t. }\left\{\begin{array}{l}
\mathbf{A}_{e} \sigma^{\mathrm{UB}}+\mathrm{A}_{\xi} s^{\mathrm{UB}}+\lambda \boldsymbol{F}=0 \\
\sigma^{\mathrm{UB}} \in \mathcal{B}, \\
\boldsymbol{s}^{\mathrm{UB}} \in \mathcal{B} .
\end{array}\right.
$$

where $\mathbf{A}_{e}$ and $\mathbf{A}_{\xi}$ are the resulting matrices after using a numerical quadrature for the integrals and imposing condition (13) 1 for all the nodal velocities. We will show in Section 3.2 that this choice is crucial for obtaining a strict upper bound.

For conic plastic criteria such as von Mises or 2D Mohr-Coulomb, and as it has been reported in $[7,10-12,17]$, the use of convenient linear transformations of the stress tensor allows to replace the set $\mathcal{B}$ by a Lorentz cone defined by,

$$
\mathcal{L}:=\left\{\boldsymbol{x} \in \mathbb{R}^{n} \mid x_{1} \geqslant\left\|\left\{x_{2}, \ldots, x_{n}\right\}\right\|\right\} .
$$

This transformation has allowed to use efficient optimisation algorithms for second order programming $[18,19]$. We note that a non-negligible computational cost reduction is gained if instead of the stress tensor $\boldsymbol{s}^{\xi, \mathrm{UB}}$, we use its projection onto direction $\boldsymbol{n}^{\xi}$, that is, the vector $\boldsymbol{t}^{\xi, \mathrm{UB}}=\boldsymbol{s}^{\xi, \mathrm{UB}} \boldsymbol{n}^{\xi}$ as a primary variable. In this case, the admissible set $\mathcal{B}$ must be replaced by an admissible set $\mathcal{B}_{t}$ for the vector field $\boldsymbol{t}^{\xi, \mathrm{UB}}$, which preserves the strictness of the bounds. It is, however, not difficult to find such new set for the common Coulomb or von Mises plastic criteria, as it is shown in [12], and indeed, we have tested that the same bounds are obtained if we use either $\boldsymbol{s}^{\xi, \mathrm{UB}}$ or $\boldsymbol{t}^{\xi, \mathrm{UB}}$ as primary variables.

We also note that the same restrictions on the variations of the material pointed out in [8] also apply here, that is, strict upper bounds cannot be guaranteed if the cohesion varies within each element. 
3.1.2. Dual optimisation problem and normality rule. Resorting to the definitions in (12), the discrete form of the dual problem in (7) may be rewritten as,

$$
\lambda^{\mathrm{UB}}=\min _{\substack{\mathrm{UB} \\ F\left(\boldsymbol{v}^{\mathrm{UB}}\right)=1}} D\left(\boldsymbol{v}^{\mathrm{UB}}\right)
$$

where $D\left(v^{\mathrm{UB}}\right)$ is the discrete form of the dissipation power in (6):

$$
\begin{aligned}
D\left(\boldsymbol{v}^{\mathrm{UB}}\right) & =\sum_{e=1}^{N_{e}} D^{e}\left(\boldsymbol{v}^{e, \mathrm{UB}}\right)+\sum_{\xi=1}^{N_{\xi}} D^{\xi}\left(\boldsymbol{v}^{\xi, \mathrm{UB}}\right), \\
D^{e}\left(\boldsymbol{v}^{e, \mathrm{UB}}\right) & =\max _{\boldsymbol{\sigma}} \int_{\Omega^{e} \mathrm{UB} \in \mathcal{B}} \boldsymbol{\varepsilon}\left(\boldsymbol{v}^{e, \mathrm{UB}}\right): \boldsymbol{\sigma}^{e, \mathrm{UB}} \mathrm{d} \Omega, \\
D^{\xi}\left(\boldsymbol{v}^{\xi, \mathrm{UB}}\right) & =\max _{s_{\xi, \mathrm{UB} \in \mathcal{B}}} \int_{\Gamma} \llbracket \boldsymbol{v}^{\xi, \mathrm{UB}} \rrbracket \bar{\otimes} \boldsymbol{n}^{\xi}: \boldsymbol{s}^{\xi, \mathrm{UB}} \mathrm{d} \Gamma .
\end{aligned}
$$

\section{After comparing the optimisation problems in (15) and (11), it is clear that because the value of} $\lambda^{\mathrm{UB}}$ is the result of minimising the dissipation power with respect to the velocities, strict bounds of $\lambda$ are achieved if the following two conditions hold:

\section{C1. The minimisation of the discrete dissipation powers defined in (16b) and (16c) enforces that} the velocity field is kinematically admissible, that is, that the normality rules are satisfied everywhere:
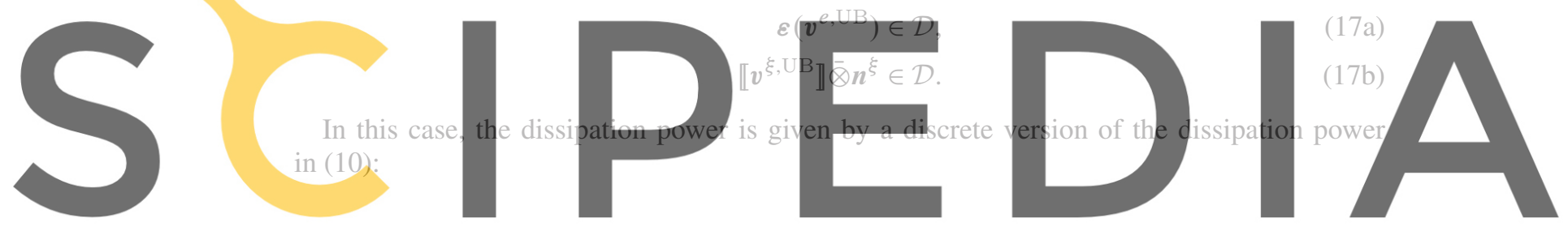

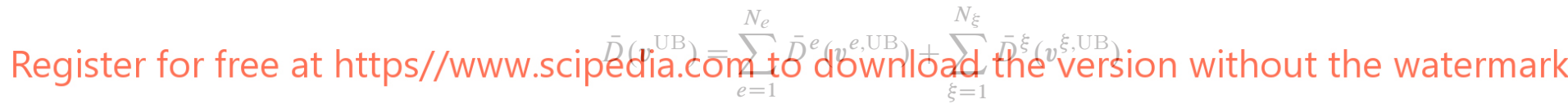

$$
\begin{aligned}
& \bar{D}^{e}\left(\boldsymbol{v}^{e, \mathrm{UB}}\right)=\int_{\Omega^{e}} k\left(\varepsilon\left(\boldsymbol{v}^{e, \mathrm{UB}}\right)\right) \mathrm{d} \Omega \\
& \bar{D}^{\xi}\left(\boldsymbol{v}^{\xi, \mathrm{UB}}\right)=\int_{\Gamma^{\xi}} k_{n}\left(\llbracket \boldsymbol{v}^{\xi, \mathrm{UB}} \rrbracket\right) \mathrm{d} \Gamma
\end{aligned}
$$

C2. The numerical integration of the discrete dissipation power $\bar{D}\left(\boldsymbol{v}^{\mathrm{UB}}\right)$ mentioned earlier yields an exact value or an upper bound of $D(\boldsymbol{v})$ in (6).

Whether the optimisation problem in (15)-(16) actually satisfies conditions $\mathrm{C} 1$ and $\mathrm{C} 2$ depends on the quadrature employed to compute the dissipation power $D\left(\boldsymbol{v}^{\mathrm{UB}}\right)$. This aspect will be analysed next.

\subsection{Numerical integration of dissipation power}

3.2.1. Elemental dissipation power. Regarding the term $D^{e}\left(v^{e, \mathrm{UB}}\right)$ in $(16 \mathrm{~b})$, we will assume that the material properties are element-wise constant. It follows that because the strain rates and stresses are constant within each element, the elemental dissipation power in (16b) is given by,

$$
D^{e}\left(\boldsymbol{v}^{e, \mathrm{UB}}\right)=A \max _{\boldsymbol{\sigma} e, \mathrm{UB} \in \mathcal{B}} \boldsymbol{\varepsilon}\left(\boldsymbol{v}^{e, \mathrm{UB}}\right): \boldsymbol{\sigma}^{e, \mathrm{UB}}=A k\left(\boldsymbol{\varepsilon}\left(\boldsymbol{v}^{e, \mathrm{UB}}\right)\right),
$$

where $A$ is the area of the element (volume in three dimensions), and the last equality follows from the fact that we are minimising $D^{e}\left(\boldsymbol{v}^{e, \mathrm{UB}}\right)$. Consequently, $D^{e}\left(\boldsymbol{v}^{e, \mathrm{UB}}\right)$ is computed exactly if just one Gauss point quadrature is employed. 
3.2.2. Dissipation power at edges. The edge dissipation power $D^{\xi}\left(\boldsymbol{v}^{\xi, \mathrm{UB}}\right)$ in $(16 \mathrm{c})$ may be expressed as follows,

$$
\begin{aligned}
D^{\xi}\left(\boldsymbol{v}^{\xi, \mathrm{UB}}\right) & =\max _{\boldsymbol{s}, \mathrm{UB} \in \mathcal{B}} \int_{\Gamma^{\xi}} \boldsymbol{v}^{\xi, \mathrm{UB}} \cdot \boldsymbol{s}^{\xi, \mathrm{UB}} \boldsymbol{n} \mathrm{d} \Gamma=\max _{\boldsymbol{s}, \mathrm{UB} \in \mathcal{B}} \boldsymbol{s}_{j}^{\xi, \mathrm{UB}} \boldsymbol{n}^{\xi} \cdot \int_{\Gamma^{\xi}} N_{i} \llbracket \boldsymbol{v}^{\xi, \mathrm{UB}} \rrbracket_{i} \mathrm{~d} \Gamma \\
& =\max _{\boldsymbol{s}, \mathrm{UB} \in \mathcal{B}} \llbracket \boldsymbol{v}^{\xi, \mathrm{UB}} \rrbracket_{i} \cdot \mathbf{M}^{i j} \boldsymbol{s}_{j}^{\xi, \mathrm{UB}} \boldsymbol{n}^{\xi},
\end{aligned}
$$

where $i, j$ are the two nodes of the edge (three nodes of the face in 3D), and $\mathbf{M}^{i j}$ is the $i j$ th component of the mass matrix, which depends on the quadrature employed. By minimising $D^{\xi}\left(\boldsymbol{v}^{\xi, \mathrm{UB}}\right)$, we are imposing the condition,

$$
\int_{\Gamma^{\xi}} N_{i} \llbracket \boldsymbol{v}^{\xi, \mathrm{UB}} \rrbracket \bar{\otimes} \boldsymbol{n} \mathrm{d} \Gamma \in \mathcal{D}, i=1, \ldots, N_{\xi} \times n_{s d},
$$

which may be interpreted as a weak form of the normality rule. However, we note that the implication

$$
\llbracket v^{\xi, \mathrm{UB}} \rrbracket_{i} \bar{\otimes} n^{\xi} \in \mathcal{D} \forall x \in \Gamma^{\xi} \Rightarrow \int_{\Gamma^{\xi}} N_{i} \llbracket v^{\xi, \mathrm{UB}} \rrbracket \bar{\otimes} n^{\xi} \mathrm{d} \Gamma \in \mathcal{D},
$$

holds, but the converse is in general not true, owing to the non-linearity of the condition in the set $\mathcal{D}_{M C}(\llbracket v \rrbracket)$ given in Equation (9).

Gauss quadrature: Polynomial functions may be integrated exactly using this quadrature, but numerical errors will be made for other integrands. Therefore, in view of the conditions in the
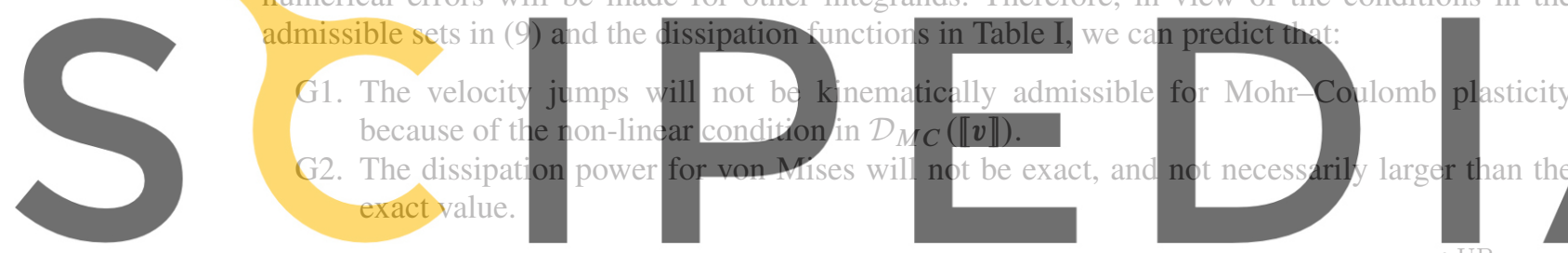

The resulting load factor, which is not necessarily an upper bound, will be denoted by $\lambda_{\mathrm{GAU}}^{\mathrm{UB}}$

Register for free at https//www.scipedia.com to download the version without the watermark

$$
\lambda_{\mathrm{GAU}}^{\mathrm{UB}}=\sum_{e=1} D^{e}\left(v^{e, \mathrm{UB}}\right)+\sum_{\xi=1} D_{\mathrm{GAU}}^{\xi}\left(v^{\xi, \mathrm{UB}}\right) .
$$

Trapezoidal rule: We will here restrain our analysis to 2D problems, although the same derivations and conclusions are valid in three dimensions.

In two dimensions, the trapezoidal rule (or two-point Lobatto quadrature) is equivalent to compute the integral from the values at the two end-points, and to use weights equal to $L / 2$, with $L$ the length of the internal edge. In this case, in view of expression (16c), the edge dissipation power is evaluated as follows,

$$
\begin{aligned}
D_{L O B}^{\xi}\left(\boldsymbol{v}^{\xi, \mathrm{UB}}\right) & =\max _{\boldsymbol{s}, \mathrm{UB} \in \mathcal{B}} \int_{\Gamma^{\xi}} \llbracket \boldsymbol{v}^{\xi, \mathrm{UB}} \rrbracket \cdot \boldsymbol{s}^{\xi, \mathrm{UB}} \boldsymbol{n} \mathrm{d} \Gamma \\
& =\max _{\boldsymbol{s}, \mathrm{UB} \in \mathcal{B}} \frac{L}{2}\left(\llbracket \boldsymbol{v}^{\xi, \mathrm{UB}} \rrbracket_{1} \cdot \boldsymbol{s}_{1}^{\xi, \mathrm{UB}} \boldsymbol{n}^{\xi}+\llbracket \boldsymbol{v}^{\xi, \mathrm{UB}} \rrbracket_{2} \cdot \boldsymbol{s}_{2}^{\xi, \mathrm{UB}} \boldsymbol{n}^{\xi}\right) \\
& =\frac{L}{2}\left(k_{n}\left(\llbracket \boldsymbol{v}_{1}^{\xi, \mathrm{UB}} \rrbracket\right)+k_{n}\left(\llbracket \boldsymbol{v}_{2}^{\xi, \mathrm{UB}} \rrbracket\right)\right)
\end{aligned}
$$

For von Mises plasticity, this expression will differ from the exact value in (16c) owing to the non-linearity of function $k_{n}$. However, the trapezoidal rule overestimates the integral of a convex function such as $\left\|\llbracket v^{\xi, \mathrm{UB}} \rrbracket\right\|=\sqrt{\llbracket v \rrbracket \cdot \llbracket v \rrbracket}$ (see Appendix A for a proof of its convexity). Furthermore, the weak form of the membership constraints for the velocity jumps turns, in this case, to the nodal enforcement of the kinematic admissibility. Therefore, from the convexity of the admissible sets, we can predict that, 
T1. The velocity jumps will be kinematically admissible at all points of the edges.

T2. The dissipation energy will be exactly computed for Mohr-Coulomb, and in general overestimated for von Mises plasticity.

We point out that because of the implication in (22) and the fact that the trapezoidal quadrature may overestimate the dissipation power, we have that,

$$
\lambda \lambda_{\mathrm{GAU}}^{\mathrm{UB}} \leqslant \lambda_{\mathrm{TRAP}}^{\mathrm{UB}},
$$

with $\lambda_{\text {TRAP }}^{\mathrm{UB}}$, the load factor obtained using the trapezoidal rule.

It has been demonstrated in [8] that the same expression $D_{\mathrm{LOB}}^{\xi}\left(\boldsymbol{v}^{\xi, \mathrm{UB}}\right)$ is attained by approximating the edge as a rectangle with two triangles, and with a thickness $\delta$ that has the limit value equal to 0 . Indeed, using the nomenclature in Figure 2(a), it can be verified that by using continuous piecewise linear velocities and piece-wise constant stresses, by setting $\llbracket v \rrbracket_{i}=\boldsymbol{v}_{i}^{b}-\boldsymbol{v}_{i}^{a}$ as the velocity jump at node $i$, and the normal $\boldsymbol{n}$ pointing from $a$ to $b$, the value of the dissipation power in the two triangles $\Omega^{a}$ and $\Omega^{b}$ is given by,

$$
D_{a b}(v)=\max _{\sigma \in \mathcal{B}} \lim _{\delta \rightarrow 0} \int_{\Omega^{a}} \sigma_{1}: \varepsilon(v) \mathrm{d} \Omega+\int_{\Omega^{b}} \sigma_{2}: \varepsilon(v) \mathrm{d} \Omega=\max _{\sigma \in \mathcal{B}} \frac{L}{2}\left(\sigma_{1} n \cdot \llbracket v \rrbracket_{1}+\sigma_{2} n \cdot \llbracket v \rrbracket_{2}\right) .
$$

Further refinements of the edge with finite width $\delta>0$ and with more elements may be contemplated. For instance, by using four elements in the interface with an additional central node (see Figure 2(b)), and making use of $\delta \rightarrow 0$ would yield the following formula for the dissipation energy at the edge:
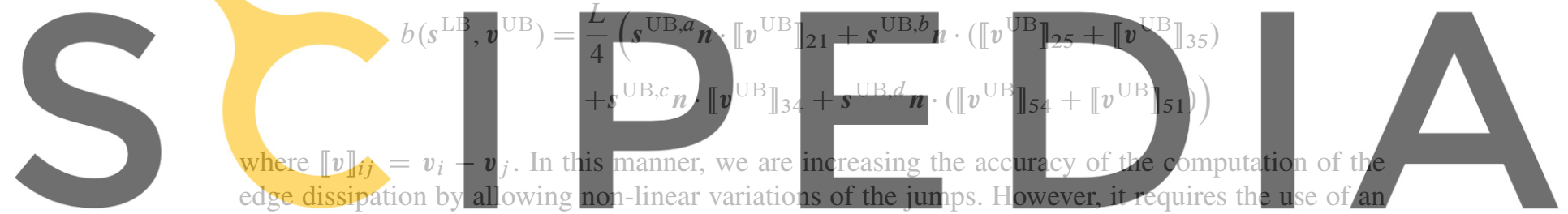

additional internal node 5 and two additional stress nodes.

Register for free at https/.www scipedia,com, to download the version without the watermark

power in (18) give rise to the integral of the square root of a second degree polynomial. More specifically, if the velocity jump is a linear function, it can be linearly parameterised using a local coordinate $\eta$ as,

$$
\llbracket \boldsymbol{v}^{\xi, \mathrm{UB}} \rrbracket(\eta)=\boldsymbol{v}_{0}+\eta \boldsymbol{v}_{1}, \eta \in[0, L],
$$

with $\boldsymbol{v}_{0}, \boldsymbol{v}_{1} \in \mathbb{R}^{2}$. Therefore, the value of $\bar{D}^{\xi}\left(\boldsymbol{v}^{\xi, \mathrm{UB}}\right)$ in (18) may be computed as follows:

$$
D^{\xi}\left(\boldsymbol{v}^{\xi, \mathrm{UB}}\right)=\int_{\Gamma^{\xi}} k_{2} \sqrt{c_{1} \eta^{2}+c_{2} \eta+c_{3}} \mathrm{~d} \Gamma .
$$

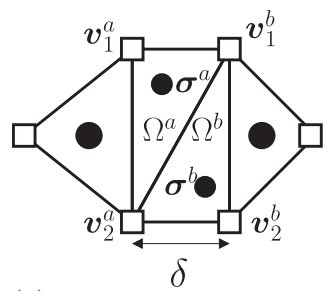

(a)

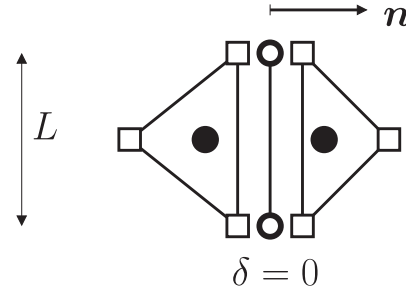

$\delta=0$

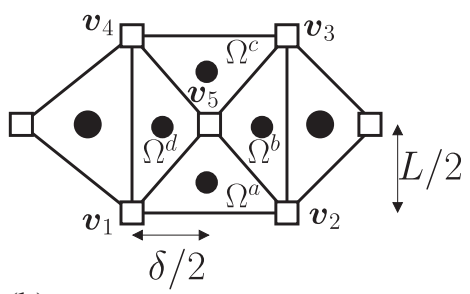

(b)

Figure 2. Piece-wise constant shape functions as a limit of two element-wise constant shape functions when the thickness $\delta$ approaches to 0 (a). Similar construction with four elements in the interface, and an additional centred node (b). 
with $c_{1}=\boldsymbol{v}_{1} \cdot \boldsymbol{v}_{1}, c_{2}=2 \boldsymbol{v}_{1} \cdot \boldsymbol{v}_{0}$ and $c_{3}=\boldsymbol{v}_{0} \cdot \boldsymbol{v}_{0}$. This integral has a primitive that can be used to compute the exact value of the dissipation power for the optimal velocity field, yielding thus a tighter bound than the trapezoidal rule. In three dimensions, we may use a composite trapezoidal quadrature, which also overestimates the integral of a convex function. Using a large enough number of sample points, the upper bound may be further reduced.

We remark that this post-process does not involve any changes in the upper bound formulation, but just a post-process of the resulting optimal velocities $\boldsymbol{v}^{\mathrm{UB}}$. Obviously, this post-process can only be applied to the velocity field resulting from the trapezoidal rule, (the Gauss quadrature does not necessarily satisfy the normality rule, and therefore the dissipation power cannot be exact).

\subsection{Error estimates}

The upper bound formulation described here can be combined with a lower bound discrete problem in order to compute error estimates at the elements, $\Delta \lambda^{e}$, and at the edges (or faces), $\Delta \lambda^{\xi}$ [12]:

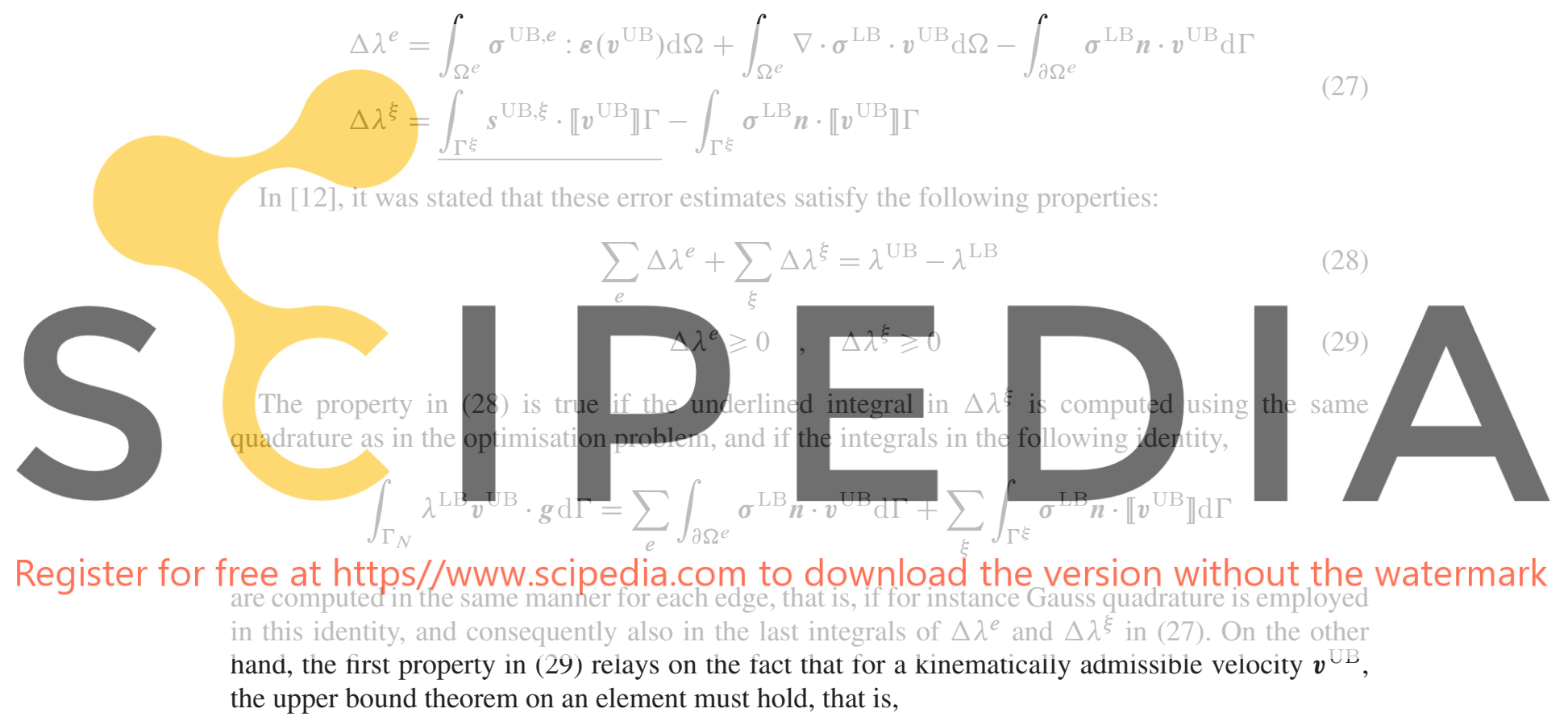

$$
\int_{\Omega^{e}} \boldsymbol{\sigma}: \boldsymbol{\varepsilon}\left(\boldsymbol{v}^{\mathrm{UB}}\right) \mathrm{d} \Omega \geqslant \int_{\Omega^{e}} \lambda^{\mathrm{LB}} \boldsymbol{f} \cdot \boldsymbol{v}^{\mathrm{UB}} \mathrm{d} \Omega+\int_{\partial \Omega^{e}} \boldsymbol{\sigma}^{\mathrm{LB}} \boldsymbol{n} \cdot \boldsymbol{v}^{\mathrm{UB}} \mathrm{d} \Gamma \forall \boldsymbol{\sigma} \in \mathcal{B}
$$

However, this is only true if the last integral is computed exactly, that is, if the two-point Gauss quadrature is employed. Summarising, the two conditions, (28) and (29) are satisfied (and strict upper bounds are obtained) if the underlined integral in (27) is computed using the trapezoidal rule, whereas all the other integrals are computed using Gauss quadrature. We have tested for the problem in Section 4.2 that if the trapezoidal rule is (incorrectly) used in the last integral of (27), it turns out that $\Delta \lambda_{\min }^{e} \approx-0.1 \Delta \lambda_{\max }^{e}$.

\section{NUMERICAL EXAMPLES}

\subsection{Rectangle with interfaces}

We have numerically tested the problem depicted in Figure 3. A rectangle is subdivided in three domains $D 1, D 2$ and $D 3$. Domain $D 2$ is subjected to a vertical tensile force $\lambda \boldsymbol{g}$, with $\boldsymbol{g}^{T}=\left\{\begin{array}{ll}0 & 1\end{array}\right\}$, and domain $D 1$ has all the velocities constrained at its left boundary. The three domains are connected at lines $\mathrm{A}-\mathrm{B}$ and $\mathrm{C}-\mathrm{D}$, where a much more restrictive plastic criteria than the one in the three domains is imposed, and therefore the failure mechanism is enforced along these lines. Two 


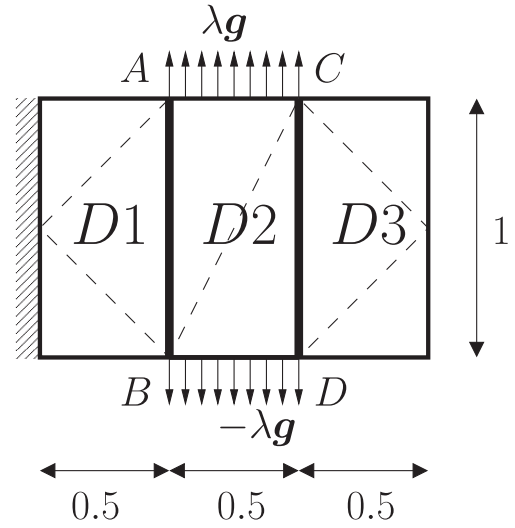

Figure 3. Geometry and boundary conditions of the rectangle problem. The dashed lines indicated the initial mesh of the geometry.

material models have been tested, von Mises and Mohr-Coulomb. The properties of the materials for the domains and the interfaces for the two models are given in Table II.

We have used the upper bound formulations described here, and the lower bound formulation in [12]. By using the strategy for the adaptive remeshing process described in [7, 12], we have refined the initial mesh depicted with dashed lines in Figure 3.

The dissipation power for the two material models are shown in Figure 4, and the evolution of the upper and lower $\lambda_{\mathrm{GAU}}^{\mathrm{UB}} \leqslant \lambda_{\mathrm{U} O \mathrm{UB}}^{\mathrm{UB}}$ ho given by the trapez

When using the the tangential velocity negligible. For this
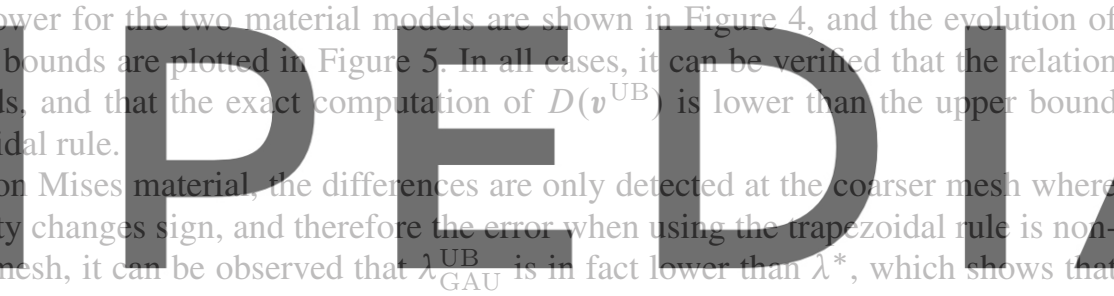

for particular configurations of the velocity jumps and when the dissipation power is concentrated

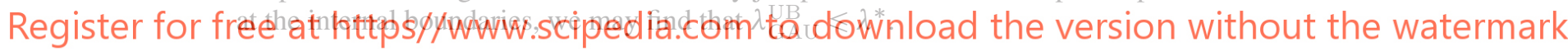

Table II. Material properties for the rectangle problem in Figure 5.

\begin{tabular}{lccc}
\hline & von Mises & \multicolumn{2}{c}{ Mohr-Coulomb } \\
\cline { 2 - 4 } & $\sigma_{Y}$ & $c$ & $\phi$ \\
\hline Domains $D 1, D 2$ and $D 3$ & $\sqrt{3}$ & 1.0 & $30^{\circ}$ \\
Interface $A-B$ and $C-D$ & $\sqrt{3} / 10$ & 0.1 & $30^{\circ}$ \\
\hline
\end{tabular}

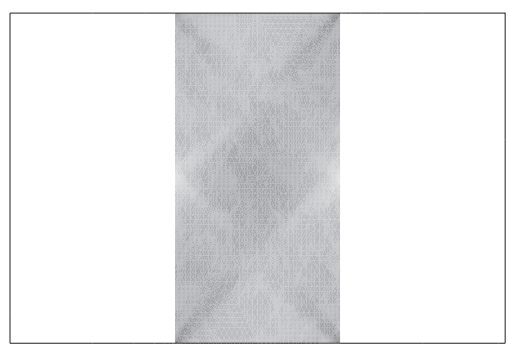

(a)

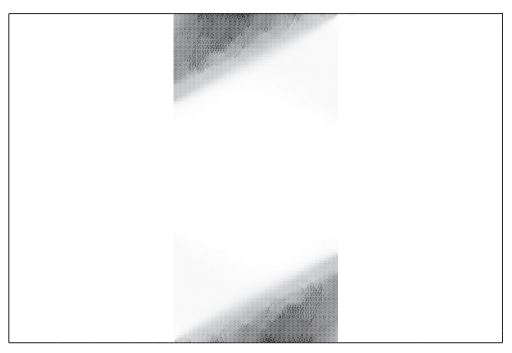

(b)

Figure 4. Contour plot of the dissipation power when using von Mises (a) and Mohr-Coulomb (b) material models, and final meshes with 17794 and 17398 elements, respectively. 

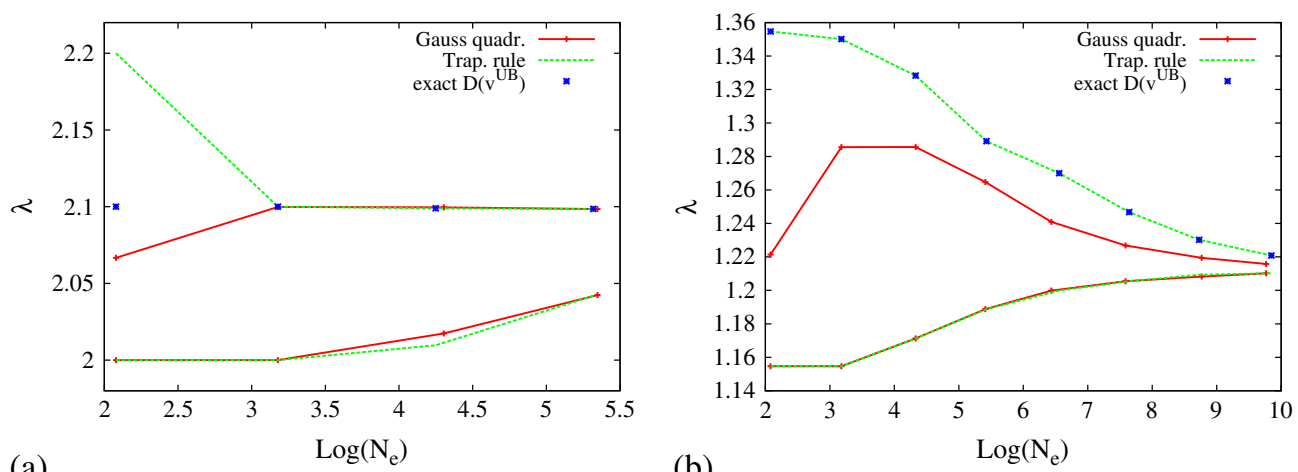

(a)

(b)

Figure 5. Evolution of the load factors for the test problem in Figure 3, using von Mises (a) and MohrCoulomb (b) material properties in Table II. The exact value of $D\left(v^{\mathrm{UB}}\right)$ is computed with the velocity field given by the formulation using trapezoidal rule.

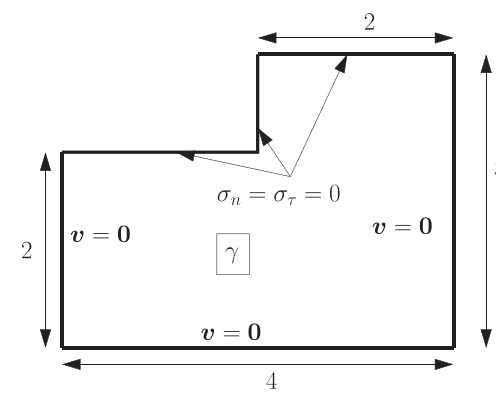

(a)

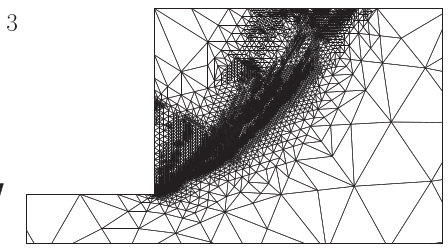

(b)

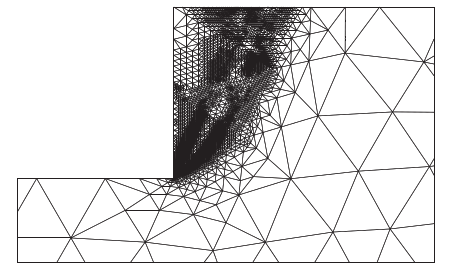

(c)

Figure 6. Geometry and boundary conditions of the vertical cut problem (a), and details of the final meshes for von Mises (b) and Mohr-Coulomb (c).

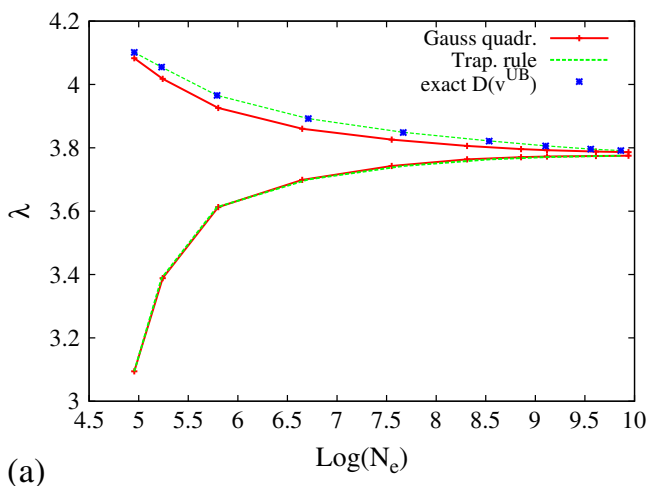

(a)

Figure 7. Evolution of the load factors for the vertical cut, using von Mises (a) and Mohr-Coulomb (b) criteria. The exact value of $D\left(v^{\mathrm{UB}}\right)$ is computed with the velocity field given by the formulation using trapezoidal rule.

For the Mohr-Coulomb material, $\lambda_{\mathrm{GAU}}^{\mathrm{UB}}$ is always above $\lambda^{*}$, but as Figure $5 \mathrm{~b}$ shows, the exact value of the dissipation power for the resulting velocity field using trapezoidal rule is for all the meshes larger than $\lambda_{G A U}^{\mathrm{UB}}$. In this case, the exact value of $D\left(v^{\mathrm{UB}}\right)$ and the upper bound $\lambda_{\mathrm{LOB}}^{\mathrm{UB}}$ coincide. 


\subsection{Vertical cut}

We have also tested the stability of the vertical cut problem, which has also been analysed for instance in $[8,9,12]$. The dimensions and boundary conditions are shown in Figure $6 \mathrm{a}$, and details of the final meshes after successive adaptive remeshing for von Mises (19177 elements) and Mohr-Coulomb (13 070 elements) are shown in Figures 6(b,c), respectively.

Figure 7 shows the evolution of the load upper and lower bound. Whereas the differences between the different quadratures are obvious, the difference between the values with exact dissipation power and the trapezoidal rule is much less noticeable. We note that the exact integration reduces, in general, the values of the upper bound by $0.01 \%$. We have also checked that if the violation of the constraints in the optimisation problem is smaller than $10^{-7}$, the properties of the gap contributions in (28) and (29) are also satisfied up to the same tolerance.

\section{CONCLUSIONS}

We have shown that despite the similarities in the formulations in $[7,8,12]$, those in [8] yield strict upper bounds of the load factor and also velocity fields that satisfy the normality rule. In contrast, the bounds in $[7,12]$ may potentially violate the strictness of the upper bound and the normality rule. We have pinpointed the source of those differences, and interpreted them in terms of the quadrature employed on the primal problem.

We have also analysed the quadratures that must be employed in the computation of the error estimates introduced in $[7,12]$ to satisfy two desired properties: positiveness and that their addition is exactly equal to the total gap.

Whereas in practice, the use of Gauss quadrature rarely yields load factors that are below the exact one, they fail to provide strict bounds of the discrete problem at hand. This discrepancy has motivated the present work. We have also suggested a post-process consisting in exactly integrating the dissipation power at the edges. In some situations, this may further reduce the upper bound formulation reported in [8], while preserving its strictness.

\section{APPENDIX A: PROOF OF CONVEXITY OF FUNCTION $\left\|\llbracket \boldsymbol{V}^{\xi, \mathrm{UB}} \rrbracket\right\|$}

We will first demonstrate the convexity of $\left\|\llbracket v^{x i, \mathrm{UB}} \rrbracket\right\|$ for $1 \mathrm{D}$ edges (2D problems), and generalise this result to $2 \mathrm{D}$ boundaries. Because of the linearity of the velocity field $\llbracket v^{\xi, \mathrm{UB}} \rrbracket$, a general velocity jump at any point on an edge $\xi$ with length $L$ may be expressed as,

$$
\llbracket \boldsymbol{v}^{\xi, \mathrm{UB}} \rrbracket(\eta)=\boldsymbol{v}_{0}+\eta \boldsymbol{v}_{1}, \eta \in[0, L],
$$

where $\eta$ is a parameter denoting the local coordinate of the point along the edge, and $\boldsymbol{v}_{1}, \boldsymbol{v}_{0} \in \mathbb{R}^{2}$. Using this notation, our aim is to demonstrate the convexity of the following function $f(\eta)$ :

$$
f(\eta)=\sqrt{\left(\boldsymbol{v}_{0}+\eta \boldsymbol{v}_{1}\right)^{T}\left(\boldsymbol{v}_{0}+\eta \boldsymbol{v}_{1}\right)} .
$$

After computing the second derivative of $f(\eta)$,

$$
f^{\prime \prime}(\eta)=\frac{\partial}{\partial \eta}\left(\frac{\left\|\boldsymbol{v}_{1}\right\|^{2} \eta+\boldsymbol{v}_{1}^{T} \boldsymbol{v}_{0}}{\left\|\llbracket \boldsymbol{v}^{\xi, \mathrm{UB}}(\eta) \rrbracket\right\|}\right)=\frac{\left\|\boldsymbol{v}_{1}\right\|^{2}\left\|\boldsymbol{v}_{0}\right\|^{2}-\left(\boldsymbol{v}_{1}^{T} \boldsymbol{v}_{0}\right)^{2}}{\left\|\llbracket \boldsymbol{v}^{\xi, \mathrm{UB}}(\eta) \rrbracket\right\|^{3}},
$$

and by resorting to Cauchy's inequality, it is clear that $f^{\prime \prime}(\eta) \geqslant 0$, and therefore $f(\eta)$ is convex.

For 3D problems, the internal boundaries between elements are triangles. In this case, the convexity of $f(\eta, \chi)=\left\|\llbracket \boldsymbol{v}^{\xi, \mathrm{UB}}(\eta, \chi) \rrbracket\right\|$ follows from the fact that the velocity jump is linear along any line on the triangular face, and therefore may be expressed according to (A.1) on such line. Consequently, because the function is convex along any line of the domain, and the domain is convex, the function $f(\eta, \chi)$ is also convex. 


\section{REFERENCES}

1. Vicente da Silva M, Ant ao A. A non-linear programming method approach for upper bound limit analysis. International Journal for Numerical Methods in Engineering 2007; 72:1192-1218.

2. Vicente da Silva M, Ant ao AN. Upper bound limit analysis with a parallel mixed finite element formulation. International Journal of Solids and Structures 2008; 45:5788-5804.

3. Pastor J, Turgeman S. Mise en oeuvre numérique des méthodes de l'analyse limite pour les matériaux de von Mises et de Coulomb standards en déformation plane. Mechanics Research Communications 1976; 3:469-474.

4. Bottero A, Negre R, Pastor J, Turgeman S. Finite element method and limit analysis theory for soil mechanics problems. Computer Methods in Applied Mechanics and Engineering 1980; 22:131-149.

5. Abdi R, de Buhan P, Pastor J. Calculation of the critical height of a homogenized reinforced soil wall: a numerical approach. International Journal for Numerical and Analytical Methods in Geomechanics 1994; 18:485-505.

6. Sloan SW, Kleeman PW. Upper bound limit analysis using discontinuous velocitiy fields. Computer Methods in Applied Mechanics and Engineering 1995; 127(5):293-314.

7. Ciria H, Peraire J, Bonet J. Mesh adaptive computation of upper and lower bounds in limit analysis. International Journal for Numerical Methods in Engineering 2008; 75:899-944.

8. Krabbenhøft K, Lyamin AV, Hjiaj M, Sloan SW. A new discontinuous upper bound limit analysis formulation. International Journal for Numerical Methods in Engineering 2005; 63:1069-1088.

9. Lyamin AV, Sloan SW. Upper bound limit analysis using linear finite elements and non-linear programming. International Journal for Numerical and Analytical Methods in Geomechanics 2002; 26:181-216.

10. Makrodimopoulos A, Martin CM. Upper bound limit analysis using simplex strain elements and second-order cone programming. International Journal for Numerical and Analytical Methods in Geomechanics 2007; 31(6):835-865.

11. Makrodimopoulos A, Martin CM. Upper bound limit analysis using discontinuous quadratic displacement fields. Communications in Numerical Methods in Engineering 2008; 24:911-927.

12. Muñoz JJ, Bonet J, Huerta A, Peraire J. Upper and lower bounds in limit analysis: adaptive meshing strategies and discontinuous loading. International Journal for Numerical Methods in Engineering 2008; 77:471-501.

13. Mihaia LA, Ainsworth M. A finite element procedure for rigorous numerical enclosures on the limit load in the analysis of multibody structures. Computer Methods in Applied Mechanics and Engineering 2009; 199:48-60.

14. Christiansen E. Handbook of Numerical Analysis, Vol IV, chapter II, Limit Analysis of Collapse States. North Holland: Amsterdam, 1996. 193-312.

15. Drucker DC. Limit analysis of two and three dimensional soil mechanics problems. Journal of the Mechanics and Physics of Solids 1953; 1:217-226.

16. Salençon J. Calcul à la Rupture et Analyse Limite. Presses de l'École Nationale des Ponts et Chaussées: Paris, 1991.

17. Krabbenhøft K, Lyamin AV, Sloan SW. Formulation and solution of some plasticity problems as conic programs. International Journal of Solids and Structures 2007; 44:1533-1549.

18. Sturm JF. Using SeDuMi 1.02, a MATLAB toolbox for optimization over symmetric cones. Optimization Methods and Software 1999; 11-12:625-653. Version 1.05 available from http://http://sedumi.ie.lehigh.edu.

19. Tütüncü RH, Toh KC, Todd MJ. Solving semidefinite-quadratic-linear programs using SDPT3. Mathematical Programming 2003; 95:189-217. Avail. http://www.math.nus.edu.sg/ mattohkc/sdpt3.html.. 